\title{
Modified Hamiltonian Algorithm for Optimal Lane Change with Application to Collision Avoidance
}

\author{
Yangyan Gao*, Mathias Lidberg ${ }^{\dagger}$ and Timothy Gordon ${ }^{\ddagger}$ \\ * University of Lincoln, Brayford Pool, Lincoln UK, e-mail: yagao@lincoln.ac.uk \\ ${ }^{\dagger}$ Chalmers University of Technology, Gothenburg, Sweden, email: mathias.lidberg@chalmers.se \\ ‡ University of Lincoln, Brayford Pool, Lincoln UK, e-mail: tgordon@lincoln.ac.uk
}

\begin{abstract}
This paper deals with collision avoidance for road vehicles when operating at the limits of available friction. For collision avoidance, a typical control approach is to: (a) define a reference geometric path that avoids collision; (b) apply low-level control to perform path following. However, there are a number of limitations in this approach, which are addressed in the current paper. First, it is typically unknown whether a predefined reference path is feasible or over-conservative. Secondly, the control scheme is not well suited to avoiding a moving object, e.g. another vehicle. Further: incorrect choice of reference path may degrade performance, fast adaptation to friction change is not easy to implement and the associated low-level control allocation may be computationally intensive. In this paper we use the general nonlinear optimal control formulation, include some simplifying assumptions and base optimal control on the minimization of an underlying Hamiltonian function. A particle model is used to define an initial reference in the form of a desired global mass-center acceleration vector. Beyond that, yaw moment is taken into account for the purpose of enhancing the stability of the vehicle. The Hamiltonian function is adapted as a linear function of tyre forces and can be minimized locally for individual wheels; this significantly reduces computational workload compared to the conventional approach of forcemoment allocation. Several combinations of actuators are studied to show the general applicability of the control algorithm based on a linear Hamiltonian function. The method has the potential to be used in future vehicle control systems across a wide range of safety applications and hence improve overall vehicle agility and improve safety.
\end{abstract}

Keywords-Collision avoidance, vehicle control, active safety, vehicle dynamics, intelligent vehicle, optimal control.

\section{Introduction}

Active safety and improving vehicle handling ability have become more and more important due to the continuing high number of accidents registered in road traffic statistics. Over the years, many car companies have successively equipped their vehicles with the most up-to-date driving assistance systems, from the earliest anti-lock braking systems (ABS), to the more recent electronic stability control (ESC) system, to adaptive cruise control (ACC) system, and to the latest collision avoidance and crash mitigation systems. For example, Volvo City Safety equipped cars have shown the effectiveness of helping the driver to avoid rear-end collisions at low speeds by automatically braking when a potential collision is detected [Distner 2007]. Similar functionality can be found in Mercedes-Benz's Pre-safe Brake and Volkswagen's Front
Assist and City Emergency Brake [Galvani 2014]. These systems use brakes only to achieve collision avoidance. The availability of multiple actuators, like individual wheel braking, active front wheel steering, active rear wheel steering and etc., enables an agile response from the vehicle, even compared to that of the most skilled human driver. Some research has been carried out around using multiple actuators for collision avoidance. In [Shah 2014], the proposed system helps the driver to steer around the obstacle by overlaying steering torque and braking individual wheels. In [Choi 2011], an emergency driving support (EDS) algorithm is developed to support the driver to avoid collision using motor driven power steering (MDPS) and electronic stability control (ESC). In [Dingle 2010], the performances of front-steer and four wheel steer vehicles are compared. It is shown that, by adding more actuators, better manoeuvrability and more agile vehicle response can potentially be achieved.

In this paper, steering and braking control are tapped, and collision avoidance is set in the context of single lane change scenario. Traditionally, collision avoidance control is based on a two-stage approach: 1) define a reference geometric path that avoids collision, 2) apply low level control to perform path following. For example, in [Shim 2012], a collision-free trajectory is determined by a motion planner based on polynomial parameterization, then a model-predictive-control (MPC)-based control system performs the path following. In [Shibata 2014], a collision avoidance method with steering control is proposed by generating a trajectory for obstacle avoidance based on application of the velocity potential field. In [Shah 2014], the optimal collision avoidance path planning was treated as a problem of finding the optimal acceleration as a function of time or path length, an optimal braking and steering control are used for path following. However, there are a number of limitations in this approach; the initial definition of the path happens as a one-time computation, and loop closure tracks that at a later time, something that can lead to a path that is too aggressive or too conservative, especially as the avoidance scenario unfolds. Furthermore, fast adaptation to friction change is not easy to implement and the associated low-level control allocation is usually computationally intensive.

To address some of the above-mentioned problems, here we develop a new approach based on steering function (rapid lane change) but using a combination of braking and steering. 
This is, however, inspired by previous work on post impact vehicle dynamics [Yang 2012], where a quasi-linear optimal controller (QLOC) is designed in the way of combining linear costate dynamics with nonlinear constraints due to tyre friction limits. Nonlinear optimal control theory then provides a semiexplicit approximation for optimal path control. This avoids a two-stage approach. Previous studies have shown applying yaw moment control may help to generate useful mass centre forces for the optimal control path. In QLOC paper, yaw moment control is achieved by optimal control theory, here a simpler alternative way of tracking desired yaw moment is proposed. The controller is designed in a hierarchical structure; first, global acceleration vector is determined using a particle model; secondly, vehicle stability control is considered by constraining yaw moment to follow a desired yaw moment profile; lastly, control allocation is achieved by minimizing Hamiltonian function at individual wheels level. Note that, the particle model used here is not for path following, instead is for QLOC integration and low-level actuation, so the motion is maximized in a general preferred direction away from possible collision.

\section{Optimal lane change strategy}

Consider a particle representation of the vehicle model with mass $m$, acted upon by friction force of magnitude $F$. We assume a planar motion with yaw motion suppressed, and use the inertial coordinate system $X_{E}, Y_{E}$ to locate the particle. The resultant force direction angle $\theta(t)$ is the control variable for the system, see Fig. 1. The coordinate origin $O$ is chosen at the vehicle's mass centre at the entry of the path. The entry velocity is not zero at the lateral direction so that the control loop can be closed during the manoeuvre. The equations of motion are

$$
\begin{aligned}
& \ddot{X}_{E}=\frac{F}{m} \cos \theta \\
& \ddot{Y}_{E}=\frac{F}{m} \sin \theta
\end{aligned}
$$

Here $F$ and $\theta$ are bounded

$$
U=\{F \in[0, \mu m g], \theta \in[0,2 \pi]\}
$$

The optimal lane change problem is formulated as a minimum terminal time optimal control problem. The objective is to find the admissible control and a feasible trajectory which minimizes the terminal time for the lane change. The cost function is

$$
J=\int_{t_{0}}^{T} 1 d t
$$

We now solve the optimal control problem using the augmented Hamiltonian system. First rewrite equation (1) in the state-space form $\dot{\boldsymbol{x}}=\boldsymbol{f}(\boldsymbol{x}, \boldsymbol{\gamma})$, or more specifically

$$
\dot{\boldsymbol{x}}=\boldsymbol{A x}+\boldsymbol{q}(\gamma), \quad \forall \gamma \in \boldsymbol{U}
$$

where the state vector $\boldsymbol{x}$ and input vector $\boldsymbol{\gamma}$ are given by

$$
\begin{aligned}
& \boldsymbol{x}=\left[\begin{array}{llll}
X_{E} & Y_{E} & \dot{X}_{E} & \dot{Y}_{E}
\end{array}\right]^{T} \\
& \gamma=\left[\begin{array}{ll}
F & \theta
\end{array}\right]^{T} \\
& \mathbf{A}=\left[\begin{array}{ll}
\mathbf{0}_{2 \times 2} & \mathbf{I}_{2 \times 2} \\
\mathbf{0}_{2 \times 2} & \mathbf{0}_{2 \times 2}
\end{array}\right] \\
& \boldsymbol{q}(\gamma)=\frac{F}{m}\left[\begin{array}{c}
\mathbf{0}_{2,1} \\
\cos \theta \\
\sin \theta
\end{array}\right]
\end{aligned}
$$

Here $\mathbf{0}_{2 \times 2}$ is the $2 \times 2$ zero matrix, $\boldsymbol{I}_{2 \times 2}$ is the $2 \times 2$ identity matrix, etc.. Introducing co-state vector $\lambda$ and Hamiltonian function $H=\lambda^{T} \boldsymbol{f}+1$, the augmented system equations are

$$
\begin{gathered}
\dot{\boldsymbol{x}}=\frac{\partial H}{\partial \boldsymbol{\lambda}} \\
\dot{\lambda}=-\frac{\partial H}{\partial \boldsymbol{x}}
\end{gathered}
$$

From (7), we obtain

$$
\dot{\lambda}=-\mathbf{A} \lambda=\left[\begin{array}{llll}
0 & 0 & -\lambda_{1} & -\lambda_{2}
\end{array}\right]^{T}
$$

The augmented equations are easily integrated to yield

$$
\lambda(t)=\left[\begin{array}{llll}
C_{1} & C_{2} & -C_{1} t+C_{3} & -C_{2} t+C_{4}
\end{array}\right]^{T}
$$

where $C_{1}, C_{2}, C_{3}$ and $C_{4}$ are integration constants.

The explicit form of the Hamiltonian function is

$$
H=\lambda_{1} x_{1}+\lambda_{2} x_{2}+\frac{F}{m}\left(\lambda_{3} \cos \theta+\lambda_{4} \sin \theta\right)+1
$$

For the free-time optimal control problem, of the form $H(T)=0$, where $T$ is the terminal time; from the form of the above equations, it then follows that $H(t)=0$ during the entire integration interval. Also, since $F$ appears linearly in equation (10); it turns out that the optimal force $F^{*}$ takes its boundary value, i.e.

$$
F^{*}=\mu m g
$$

The optimality condition is then given by

$$
\begin{gathered}
\frac{\partial H}{\partial \theta}=\frac{F}{m}\left(\lambda_{3} \sin \theta-\lambda_{4} \cos \theta\right)=0 \\
\tan \theta^{*}=\frac{\lambda_{4}}{\lambda_{3}}
\end{gathered}
$$

We now consider the terminal conditions for optimal control. Assuming the combination of braking and steering strategy is successful, the lane change is considered completed when the global lateral velocity reaches zero. Therefore, the particle is transferred to a lane parallel to the global $\underline{X}_{E}$-axis at a distance $d_{0}$ away from the original lane. Thus, the complete set of boundary conditions is: 


$$
\begin{array}{ll}
X_{E}(0)=0 & \lambda_{1}(T)=0 \\
Y_{E}(0)=0 & Y_{E}(T)=d_{0}, \lambda_{2}(T)=v_{2} \\
\dot{X}_{E}(0)=v_{0} \cos \theta_{0} & \lambda_{3}(T)=0 \\
\dot{Y}_{E}(0)=v_{0} \sin \theta_{0} & \dot{Y}_{E}(T)=0, \lambda_{4}(T)=v_{4}
\end{array}
$$

where $v_{2}$ and $v_{4}$ are constants to be determined so that $Y_{E}(T)=d_{0}$ and $\dot{Y}_{E}(T)=0$. From $\lambda_{1}(T)=0$, we find $C_{1}=0$, and then $\lambda_{3}=C_{3}=0$ from $\lambda_{3}(T)=0$. From (13), we obtain $\theta^{*}=\frac{\pi}{2}$ for $t<t_{s}, \frac{3 \pi}{2}$ for $t>t_{s}$ where $t_{s}$ is a switching time which the resultant force acts in the lateral direction, and the optimal control input [ $\left.F^{*}(t) \theta^{*}(t)\right]^{T}$ is found to be

$$
\begin{aligned}
& F^{*}(t)=\mu m g \\
& \theta^{*}= \begin{cases}\frac{\pi}{2} & t<t_{s} \\
\frac{3 \pi}{2} & t>t_{s}\end{cases}
\end{aligned}
$$

where the switching time corresponds to $\lambda_{4}\left(t_{s}\right)=0$. Further, assuming the initial lateral velocity is zero, the overall time for the lane change is found to be

$$
T^{*}=2 \sqrt{\frac{d_{0}}{\mu g}}
$$

\section{Vehicle model}

Beyond the very simple particle, we use a nonlinear vehicle model for simulation. It is a 3-degree-of freedom (DOF) planar two-track model with three states $v_{x}, v_{y}, \dot{\psi}$, relative to the vehicle $x-y$ coordinates. This model is suitable for the application of active brake controls, front wheel steering controls and four wheel steering controls. The equations of motion are as follows:

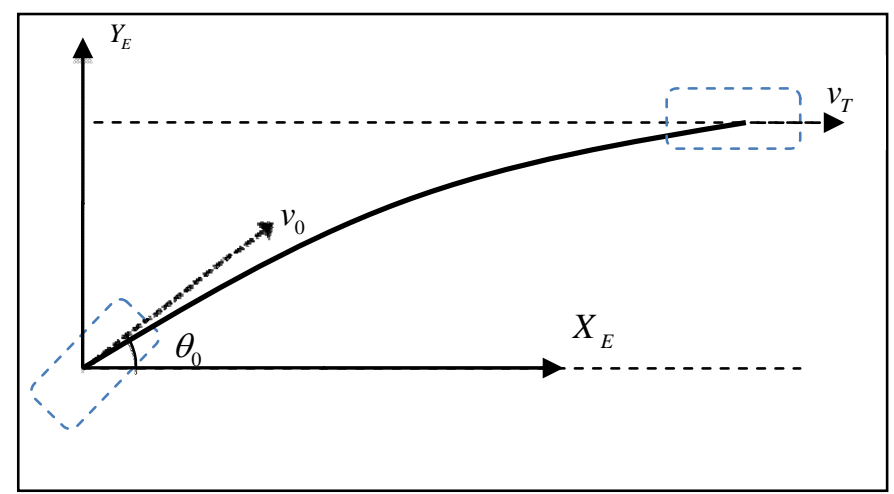

Figure 1. Kinematics of the rapid lane-change used for the collision avoidance maneuvre.

$$
\begin{gathered}
m \cdot\left(\dot{v}_{x}-v_{y} \cdot \dot{\psi}\right)=F_{x 1}+F_{x 2}+F_{x 3}+F_{x 4} \\
m \cdot\left(\dot{v}_{y}+v_{x} \cdot \dot{\psi}\right)=F_{y 1}+F_{y 2}+F_{y 3}+F_{y 4} \\
I_{x x} \cdot \ddot{\psi}=l_{a} \cdot\left(F_{y 1}+F_{y 2}\right)-l_{b} \cdot\left(F_{y 3}+F_{y 4}\right) \\
+l_{c} \cdot\left(F_{x 2}-F_{x 1}\right)+l_{c} \cdot\left(F_{x 4}-F_{x 3}\right)
\end{gathered}
$$

The tyre forces $\left(F_{x i}, F_{y i}\right)$ are defined via a standard Pacejka model, data for which are presented below. Furthermore, load transfer is included and tyre forces are likewise sensitive to these vertical loads. Similar models are used and detailed in references [Longoria 2009], [Fredriksson 2004]. Vehicle axes are shown in Fig. 2, and subscripts such as $F_{x i}$ correspond to the corner labels shown. The nonlinear tyre model characteristics are crucial to solving the limit handling problem; relevant tyre force curves are presented below.

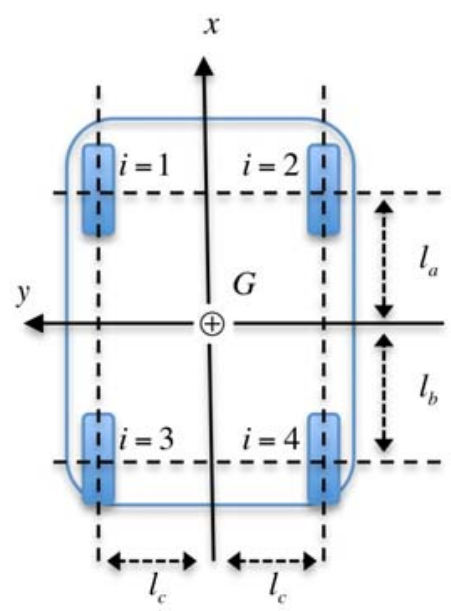

Figure 2. Layout of vehicle including axes

\section{Control allocation in limit handling via the hamiltonian function}

The aim now is to convert acceleration demands, obtained from the particle model above, into actuator control signals: front wheel steering angles or four wheel steering angles and individual braking torques. One way to achieve this is via control allocation algorithms [Longoria 2009], [Fredriksson 2004]. Such algorithms typically use constrained nonlinear optimization methods to determine feasible (and in some sense optimal) inputs at the actuators to achieve the required vehiclelevel forces and moments $\left[F_{x}, F_{y}, M_{z}\right]^{T}$.This is a computationally intensive process, since it uses nonlinear models of all four tyres simultaneously and an alternative approach was developed in QLOC papers [Yang 2012], [Yang 2014] which includes an optimal trade-off between global accelerations and yaw moments. This was calculated using a linearized model to approximate the co-state functions. Here we adopt a simpler approach that is nonetheless motivated by the QLOC formulation. Let $\mathbf{a}^{d}=\left[a_{x}^{d}, a_{y}^{d}\right]^{T}$ be the desired mass centre acceleration vector, which we assume to be on the limits 
of friction. In the absence of any yaw moment target this is achieved by minimizing the following linear function $H=p_{x} F_{x}+p_{y} F_{y}$ where we define $\mathbf{p}$ as a unit vector in the direction opposing the desired acceleration, $\mathbf{p}=-\mathbf{a}^{d} /\left|\mathbf{a}^{d}\right| . H$ is to be minimized subject to friction constraints for the whole vehicle. On the other hand, yaw moments may be applied to adjust body sideslip angle and enhance the ability of the vehicle to generate useful mass-centre forces within some control-planning horizon. In the QLOC formulation this was determined via optimal control theory, while here we find an alternative approach to target a desired yaw moment $M_{z}$. To achieve this, the Hamiltonian function is adapted to

$$
H=p_{x} F_{x}+p_{y} F_{y}+\lambda M_{z}
$$

where $\lambda$ plays a role similar to a Lagrange multiplier, in order to constrain the yaw moment. In the following we determine a desired yaw moment $M_{z}^{d}$ and $\lambda$ is to be adapted to track this value:

$$
\lambda \rightarrow \lambda+\Delta \cdot \operatorname{sgn}\left(M_{z}-M_{z}^{d}\right)
$$

where here $\Delta$ is chosen as a fixed increment per controller time step. The key point is that this is a linear function of tyre forces and may be minimized independently via the forces acting at each individual tyre. We now formulate this by resolving $H$ as a sum over longitudinal and lateral tyre forces.

As mentioned, the control objective is formulated as $H \rightarrow \min$. Any coordinate system may be used in equation (18), but here we adopt vehicle coordinates:

$$
\begin{aligned}
& F_{x}^{v}=\sum_{i} F_{x i}^{v} \\
& F_{y}^{v}=\sum_{i} F_{y i}^{v}
\end{aligned}
$$

where the superscript $v$ indicates the coordinate system. The yaw moment is expressed as

$$
M_{z}=\sum_{i}\left(x_{i} F_{y i}^{v}-y_{i} F_{x i}^{v}\right)
$$

We also consider global coordinates $(g)$ and also "tyre" coordinates $(t)$ aligned with the wheels which may be steered relative to the vehicle through angle $\delta_{i}$; see Fig. 3 .

From the figures the path angle $\phi$ is related to the vehicle yaw angle $\psi$ and sideslip angle $\beta$ via the equation:

$$
\psi=\phi+\beta
$$

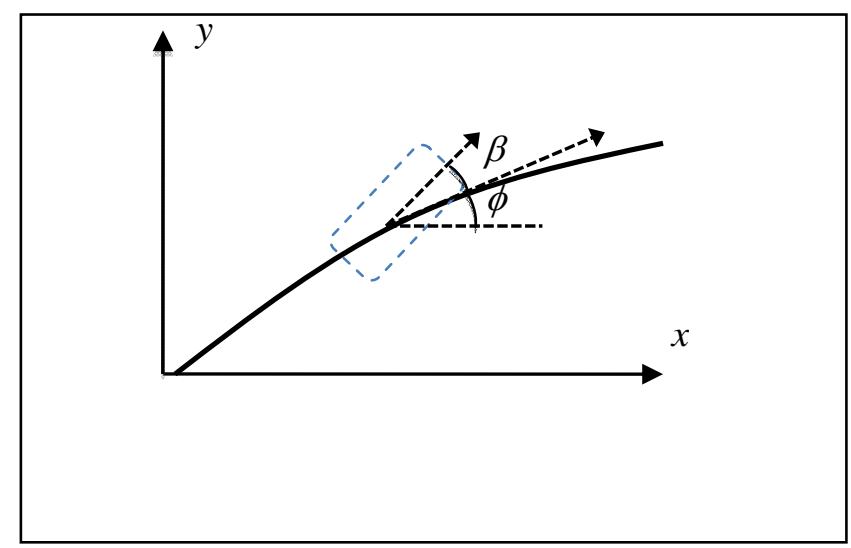

Figure 3. Path and yaw angle

Further, the global "yaw" angle $\theta_{i}$ of an individual wheel is given by

$$
\theta_{i}=\psi+\delta_{i}
$$

where $\delta_{i}$ is the steer angle relative to the vehicle. In global axes, the individual wheel forces $\mathbf{F}_{i}^{g}=\left[F_{x i}^{g}, F_{y i}^{g}\right]^{T}$ are related to the corresponding forces $\mathbf{F}_{i}^{t}$ in tyre coordinates via

$$
\mathbf{F}_{i}^{g}=\boldsymbol{R}\left(\theta_{i}\right) \mathbf{F}_{i}^{t}
$$

where $\boldsymbol{R}\left(\theta_{i}\right)$ is the 2D rotation matrix defined by

$$
\boldsymbol{R}(\theta)=\left[\begin{array}{cc}
\cos \theta & -\sin \theta \\
\sin \theta & \cos \theta
\end{array}\right]
$$

In the Hamiltonian function, both $\mathbf{p}$ and $\mathbf{F}$ are assumed to be in global coordinates. It is convenient to convert to vehicle coordinates, $H=\mathbf{p}^{v} \cdot \mathbf{F}^{v}+\lambda M_{z}$, where $\mathbf{p}^{v}=\boldsymbol{R}(\psi)^{T} \mathbf{p}^{g}$. Then we decompose into component wheel forces:

$$
H=\sum_{i} H_{i}
$$

where

$$
\begin{aligned}
H_{i} & =p_{x}^{v} F_{x i}^{v}+p_{y}^{v} F_{y i}^{v}+\lambda\left(x_{i} F_{y i}^{v}-y_{i} F_{x i}^{v}\right) \\
& =\left(p_{x}^{v}-\lambda y_{i}\right) F_{x i}^{v}+\left(p_{y}^{v}+\lambda x_{i}\right) F_{y i}^{v} \\
& \equiv \tilde{p}_{x i}^{v} F_{x i}^{v}+\tilde{p}_{y i}^{v} F_{y i}^{v} \\
& =\tilde{\mathbf{p}}_{i}^{v} \cdot \mathbf{F}_{i}^{v}
\end{aligned}
$$

Finally, converting to local tyre forces via the steering angles, $\mathbf{F}_{i}^{v}=\boldsymbol{R}\left(\delta_{i}\right) \mathbf{F}_{i}^{t}$, we obtain

$$
H_{i}=\tilde{\mathbf{p}}_{i}^{t} \cdot \mathbf{F}_{i}^{t} \rightarrow \min
$$

where $\tilde{\mathbf{p}}_{i}^{t}=\boldsymbol{R}\left(\delta_{i}\right)^{T} \tilde{\mathbf{p}}_{i}^{v}$ are the linear tyre force coefficients of the "local Hamiltonian" function. For online implementation this greatly simplifies the analysis, since the individual tyre forces are effectively decoupled. There is a minor interaction: as in-plane tyre forces are varied the vertical loads are 
affected, but in a real vehicle the effect is moderated by the suspension system and vertical loads can be estimated independently of the braking and cornering forces.

\section{Control of tyre forces yaw moments}

In the above it has been shown that, based on a linear Hamiltonian, control force allocation at the vehicle level is reduced to function minimization at the local wheel level. The tyre model we use here is Pacejka magic formula tuned to representative tyre data given in CarSim D Class Sedan lateral force map [Mechanical Simulation 2005]- Fig. 4.

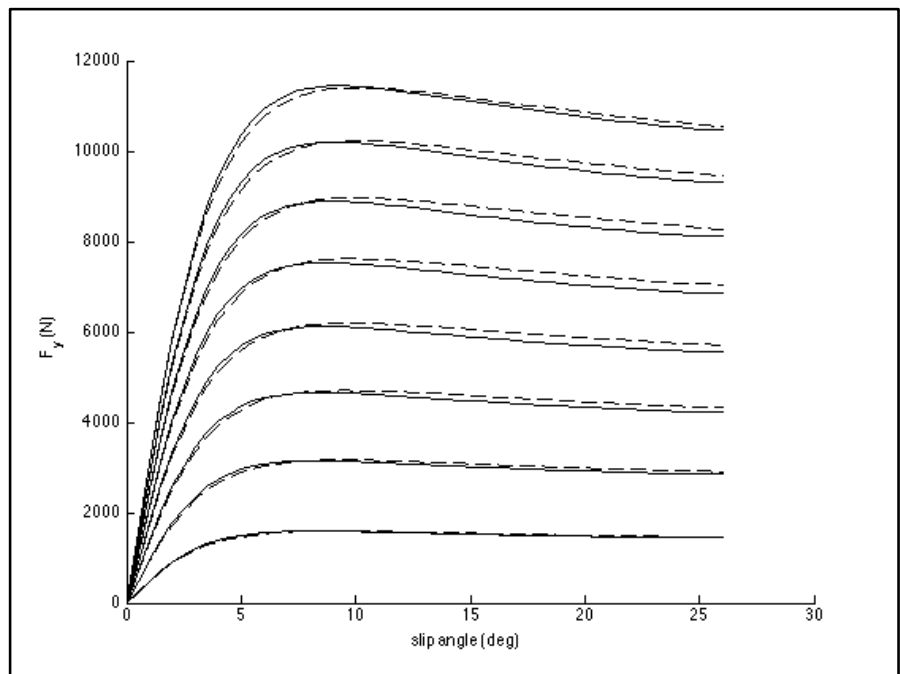

Figure 4. Nonlinear tyre forces: lateral force as a function of slip angle at 8 different vertical loads [solid: CarSim data, dashed: Pacejka 'magic formula' model.

For $H$ minimization we start with the form of equation (28). It is convenient to define the $H$-angle $v$ corresponding to the ratio of the components $\tilde{\mathbf{p}}_{i}^{t}: \tan v_{i}=\tilde{p}_{i 2}^{t} / \tilde{p}_{i 1}^{t}$ so that, equivalent to (28) we have

$$
\hat{H}_{i}=\cos v_{i} \cdot F_{i 1}^{t}+\sin v_{i} \cdot F_{i 2}^{t} \rightarrow \min
$$

The following figure, Fig. 5, shows a series of curves representing the range of forces available due to changes in braking torque, each at a given slip angle, with terminal value corresponding to a locked wheel. Lines of constant $\hat{H}$ are shown dotted. Stars show the points of minimum $\hat{H}$. The thicker line shows an example with modest slip angle ( $\alpha=0.25^{\circ}$ ) in which case the optimal $\left(^{*}\right.$ ) point is close to that targeted by an antilock braking system (near maximum braking force). On the other hand, for curves in the upper part of the plot, $\hat{H}$ is minimized at full braking torque, i.e. with a locked wheel.

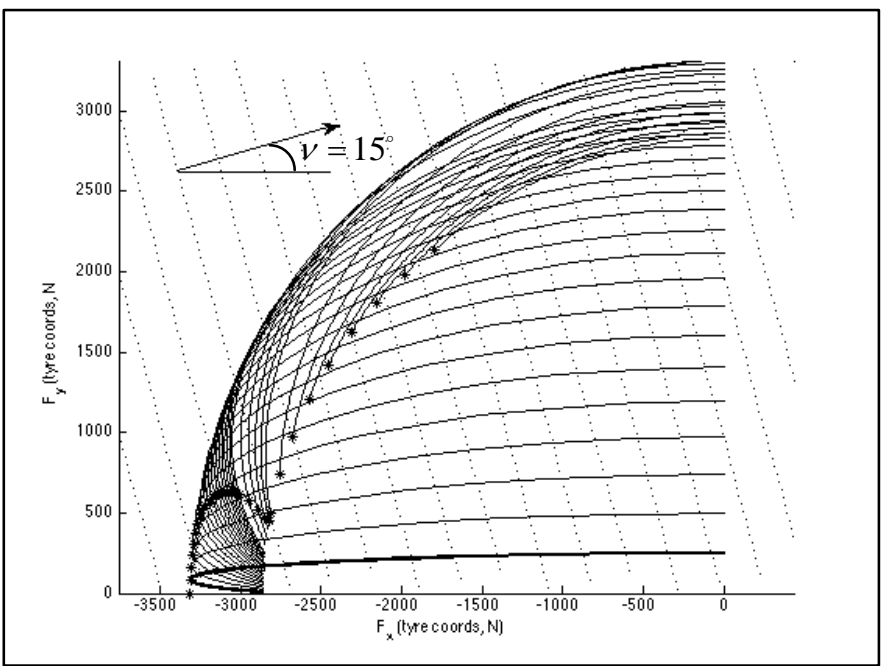

Figure 5. Force map in tyre coordinates: each solid curve represents the range of forces available due to changes in braking torque for a given slip angle, with terminal value corresponding to a locked wheel. Lines of constant $\hat{H}$ are shown dotted. Stars show the point of minimum $\hat{H}$.The thicker line shows an example with $\alpha=0.25^{\circ}$.

For closed-loop control we assume that brake forces can be rapidly changing, so the star $(*)$ point can be rapidly obtained. Further, the slip angle can be increased or decreased by (i) actively controlling the steer angle, or (ii) changing the body sideslip angle of the entire vehicle. For the example shown, $v=15^{\circ}$, it is clear that the minimizing value of $\hat{H}$ increases with slip angle, so for this wheel we should steer to reduce $\alpha$ if possible. For individual wheel steering (i) this is certainly possible, while for a single steered axle we need to consider the tradeoff between the two steered wheels. And for body sideslip control the tradeoff is for all four wheels.

More formally we assume that the tyre model is adequately known to be used in open-loop fashion, so that at a given slip angle a one-dimensional search is carried out to minimize $\hat{H}_{i}$ at each wheel. For simplicity in this analysis we assume wheel angular accelerations are sufficiently small that applied wheel torque $T_{w}$ is in equilibrium with the longitudinal tyre force: $T_{w} \approx F_{x}^{t} \cdot R_{w}$ (where $R_{w}$ is the rolling radius of the wheel/tyre). For the current slip angle, the corresponding curve in Fig. 5 determines the required value of $F_{x}^{t}$ and hence the braking torque $T_{w}$. (Note: for active driving torque control, e.g. via active differentials or individual wheel motors, we can relax this constraint). Then via a small perturbation $\pm \varepsilon$ around the 'star' point we estimate

$$
\frac{\partial H_{i}}{\partial \alpha_{i}} \approx \frac{H_{i}\left(\alpha_{i}+\varepsilon\right)-H_{i}\left(\alpha_{i}-\varepsilon\right)}{2 \varepsilon}
$$

Note that we revert to the non-normalized Hamiltonian components, since relative magnitudes are significant when evaluating the trade-off between different wheels. Now it is easy to show that when the vehicle sideslip angle $\beta$ is small we have 


$$
\alpha_{i}=\delta_{i}+\beta-v_{x}^{-1} \dot{\psi} x_{i}
$$

In the following we assume the collision avoidance system has control of the front steering angles $\left(\delta_{1}=\delta_{2}=\delta\right.$ ) and the four individual wheel braking torques $T_{w i}$. Coordination of slip angles at the front axle is via the common steer angle:

$$
\begin{aligned}
H_{\delta} & \equiv \frac{\partial H}{\partial \delta}=\frac{\partial H_{1}}{\partial \alpha_{1}} \cdot \frac{\partial \alpha_{1}}{\partial \delta}+\frac{\partial H_{2}}{\partial \alpha_{2}} \cdot \frac{\partial \alpha_{2}}{\partial \delta} \\
& =\frac{\partial H_{1}}{\partial \alpha_{1}}+\frac{\partial H_{2}}{\partial \alpha_{2}}
\end{aligned}
$$

using equation (30), assuming an effective rate limit $k_{\delta}$ for the front axle steering actuator [Yang 2014] we locally reduce the value of $H$ via the control law

$$
\dot{\delta}=\left\{\begin{array}{cc}
-k_{\delta} \operatorname{sgn}\left(H_{\delta}\right) & \text { if }\left|H_{\delta}\right|>\text { tol } \\
0 & \text { otherwise }
\end{array}\right.
$$

Body sideslip control is defined in a similar fashion, though via a dynamic degree of freedom, namely the yaw motion of the vehicle:

$$
H_{\beta} \equiv \frac{\partial H}{\partial \beta}=\sum_{i} \frac{\partial H_{i}}{\partial \alpha_{i}} \cdot \frac{\partial \alpha_{i}}{\partial \beta}=\sum_{i} \frac{\partial H_{i}}{\partial \alpha_{i}}
$$

Again using equation (30), similar to equation (33) we define a target sideslip rate based on the requirement to minimize $H$

$$
\dot{\beta}_{H}=\left\{\begin{array}{cc}
-k_{\beta} \operatorname{sgn}\left(H_{\beta}\right) & \text { if }\left|H_{\beta}\right|>\text { tol } \\
0 & \text { otherwise }
\end{array}\right.
$$

Yaw stability may also be required, to avoid increasing sideslip angle $\beta$ beyond some threshold value $\beta_{2}$, typically no more than around $5^{\circ}$ though it is not clear a priori that an additional criterion is needed. It is quite easy to introduce a saturation condition of the form

$$
\dot{\beta}_{d}=\left\{\begin{array}{cc}
-k_{\beta} \operatorname{sgn}(\dot{\beta}) & \text { if }|\beta|>\beta_{2} \\
0 & \text { if }|\beta|>\beta_{1} \cap \beta \dot{\beta}_{H}>0 \\
\dot{\beta}_{H} & \text { otherwise }
\end{array}\right.
$$

where $0<\beta_{1}<\beta_{2}$. While the switching conditions are somewhat ad-hoc and require separate definition of threshold parameters such as $\left(k_{\beta}, \beta_{1}, \beta_{2}\right)$, each parameter has a direct meaning for controller design and such rules are typical of traditional control laws for body sideslip control [Kiencke 2005]. Note however that the current analysis is for handling control under highly nonlinear operating conditions, right at the friction limits.

To complete the yaw moment control we need a process for choosing the desired $M_{z}$ and adapting the parameter $\lambda(t)$ to determine a preferred or optimal trade-off between controlling the trajectory of the vehicle mass centre and adjusting the orientation to improve body sideslip angle (and hence side forces at un-steered wheels). In [Longoria 2009] this parameter was determined via nonlinear optimal control theory. In the present work we formulate a simpler algorithm as follows: from equation (22) we determine the desired yaw rate

$$
\dot{\psi}^{d}=\dot{\phi}+\dot{\beta}^{d}
$$

$\dot{\beta}^{d}$ is determined above and $\dot{\phi}$ is related to path curvature, and is found from the acceleration in path coordinates

$$
m v \dot{\phi}=m a_{y}^{p}=F_{y}^{g} \cos \phi-F_{x}^{g} \sin \phi
$$

$v$ being vehicle speed and $F^{g}$ being the force components determined from the tyre model above. Yaw moments are applied to track the desired yaw rate, most simply via a first order control law

$$
\tau \frac{d \dot{\psi}}{d t}=\dot{\psi}^{d}-\dot{\psi}
$$

hence the desired yaw moment is

$$
M_{z}^{d}=\tau^{-1} I_{z z}\left(\dot{\psi}^{d}-\dot{\psi}\right)
$$

where $I_{z z}$ is the yaw moment of inertia.

\section{Case 1: front steer with four wheel brakes}

In Case 1, we consider a vehicle with front steer and individual wheel braking ( 5 actuators) controlled by the above Hamiltonian controller. Initially we choose $\Delta=0.15$ as a fixed increment for $\lambda$. A lane change is executed starting from a vehicle speed of $20 \mathrm{~m} / \mathrm{s}$. Fig. 6 shows the path of the vehicle, Fig. 7 gives the accelerations in vehicle coordinates and finally Fig. 8 presents the effect of adapting $\lambda$ to track the desired yaw moment. The lane change is seen to be successfully executed within a clock time $t=3.5$ seconds, i.e. 1.5 seconds from the start of the lane change. This compares with 1.2 seconds for the ideal particle. The results do not necessarily prove the optimality of the controller, but the magnitude of the mass-centre acceleration vector is close to the friction limit, the lane change is faithfully executed using automated and coordinated control of front steering and individual wheel braking, and the algorithm is both general and incorporates all existing actuator constraints (e.g. no rear steering, equal front steering left/right, no driving torque). Fig. 8 also shows that by adapting $\lambda$ it is possible to control yaw moments and actually achieve tight control of body sideslip this is suggested in Fig. 6, and the actual peak is within \pm 4 degrees. 
Figure 6. Lane Change at $20 \mathrm{~m} / \mathrm{s}$ based on the optimal particle motion of Section II (vertical axis shown in $\mathrm{m}$ ).
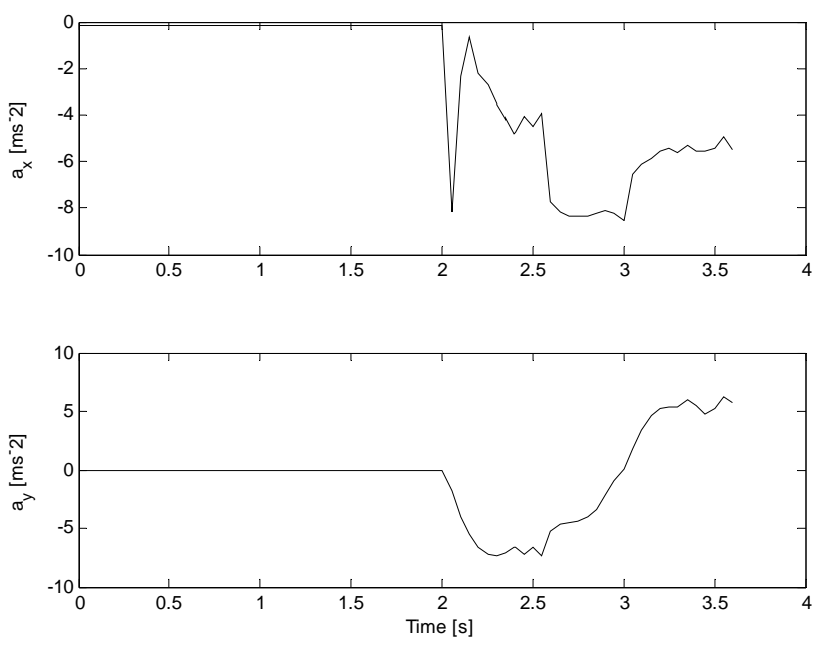

Figure 7. Case 1: Accelerations - lane change initiated at time $t=2 \mathrm{sec}$.

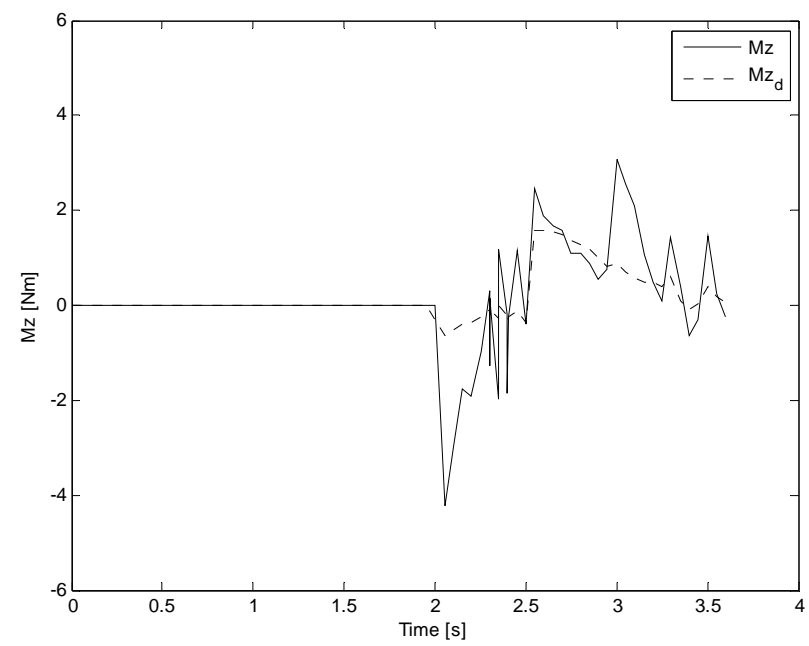

Figure 8. Case 1:Yaw Moment tracking, actual (solid) vs. desired (dashed).

The initial choice of $\Delta$ is tested now, based on how well adapting $\lambda$ will track the desired yaw moment, repeating the lane-change simulation for a range of values $\Delta$ at regular increments; from Fig. 9, a small range of values around
$\Delta=0.15$ show similar tracking performances which suggests $\Delta=0.15$ is a reasonable value of increment for $\lambda$. We also need to take account of delays in switching the sign and magnitude of the lateral acceleration, due to tire relaxation and vehicle inertia; this is clear from the lower plot of Fig. 7. Hence we need to prescribe the lane change trigger position which needs to ensure that there is a lateral motion equal to a full lane width on completion of the maneuver. From Fig. 10, we can see, for an ideal particle, lane change trigger position is at the half of the lane width, while, for a more realistic vehicle, the lane change maneuver needs to be taken at an earlier stage to compensate for the mentioned time delays; in this case the lane trigger position is chosen at 0.2 , this a normalized value with respect to lane width $W=3.5 \mathrm{~m}$; so the lane change finishes at the center of the target lane.

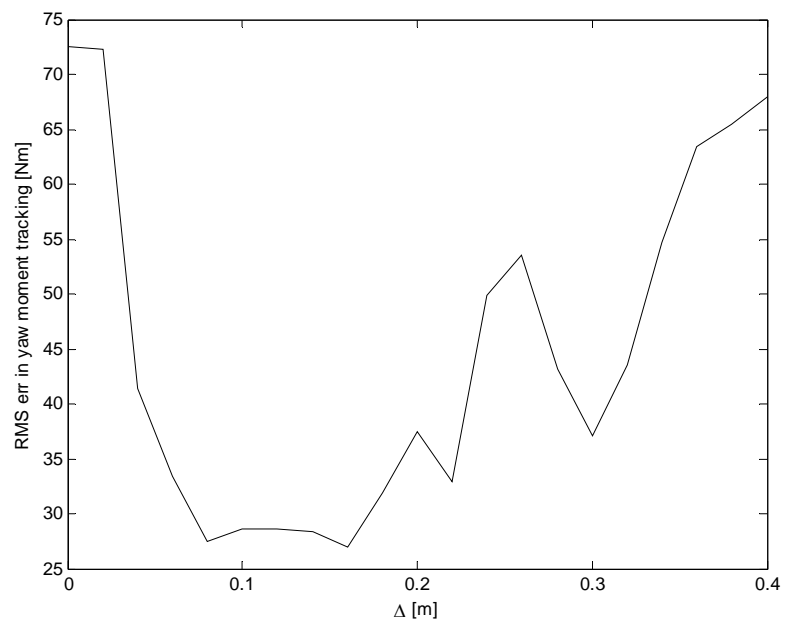

Figure 9. Optimal choice of $\Delta$ : RMS error in yaw moment tracking vs. $\Delta$

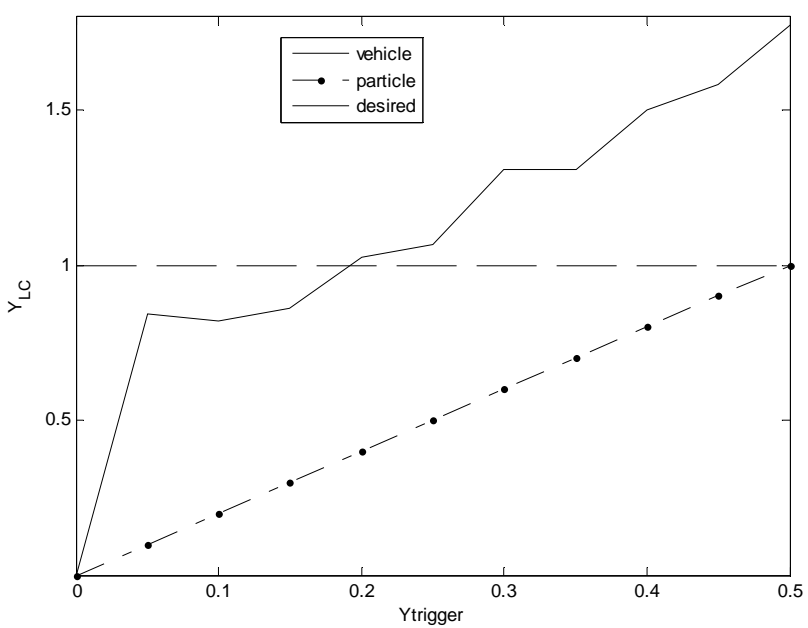

Figure 10. Normalized lane change trigger position ( $\left.Y_{\text {trigger }}\right)$ vs. Normalized lateral deviation $\left(\mathrm{Y}_{\mathrm{LC}}\right)$ - intersection between horizontal line and solid line suggests the lane change trigger position. Normalization is with respect to lane width $\mathrm{w}=3.5 \mathrm{~m}$. 


\section{Case 2: 4 wheel steering and 4 wheel braking}

In this case, we add the option for rear wheel steering (6 actuators). Going through a similar process to the above, we determine $\Delta=0.12$ and $Y_{\text {trigger }}=0.25$. It turns out that with both front and rear steering, the vehicle has a more agile response, associated with reduced yaw angle but with larger peak body sideslip angle at 10 degrees. The results are shown in Fig. 1112.
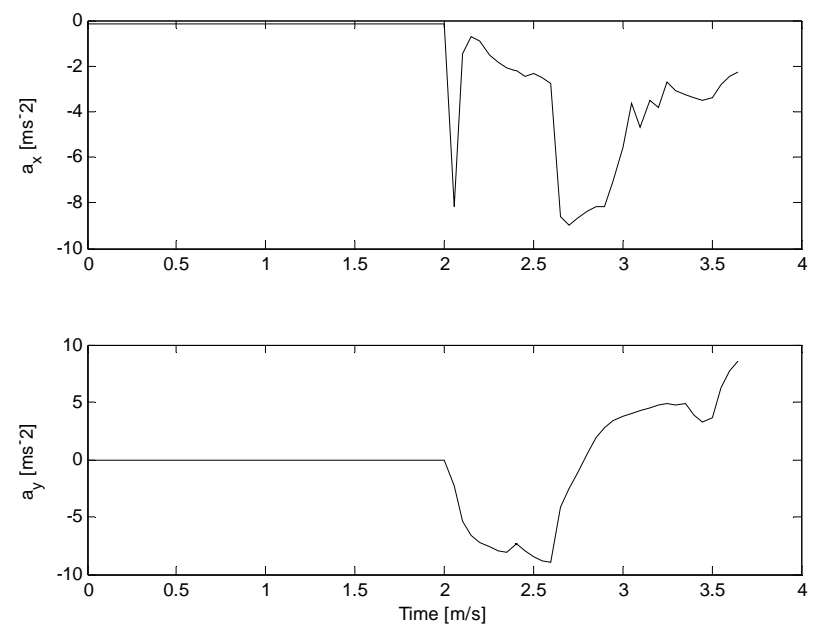

Figure 11. Case 2: Accelerations - lane change initiated at time $t=2$ sec.

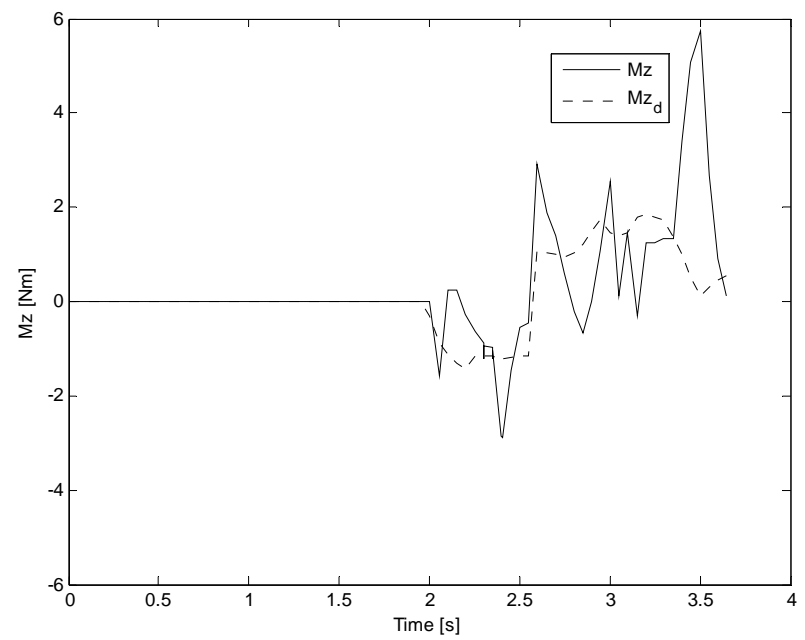

Figure 12. Case 2:Yaw Moment tracking, actual (solid) vs. desired (dashed).

\section{Case 3: 4 wheel braking only}

In this case, we do not allow any steering at all, hence with only 4 individual wheel braking; as above we determine acceptable parameters: $\Delta=0.1$ and $Y_{\text {trigger }}=0.5$. In this case the agility is greatly reduced: the vehicle takes 3.9 seconds to finish the lane change; this is much slower than previous two cases since the lane change manoeuvre is achieved only by differential braking and the vehicle speed is greatly reduced during the manoeuvre. Here peak body sideslip is controlled within \pm 4 degrees, again indicating a high degree of yaw moment control and yaw stability. The results are shown in Fig. 13-14. Recall that we assume fast (instantaneous) control of the brake forces, and for the case without steering this leads to a high degree of brake chatter, similar to that seen in sliding mode control. While lateral accelerations are suppressed in favor of longitudinal deceleration (Fig. 13), seemingly satisfactory performance is achieved and the lane change is completed.
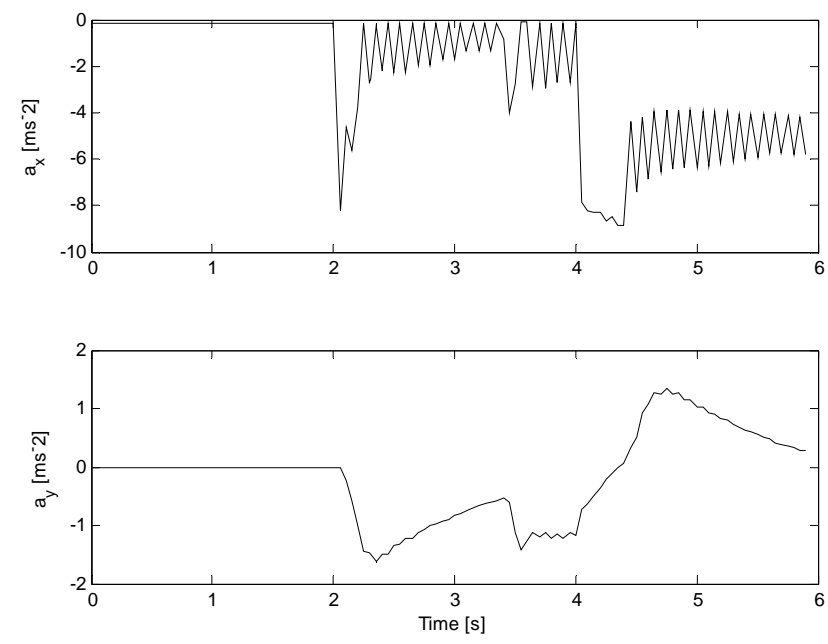

Figure 13. Case 3: Accelerations - lane change initiated at time $t=2$ sec.

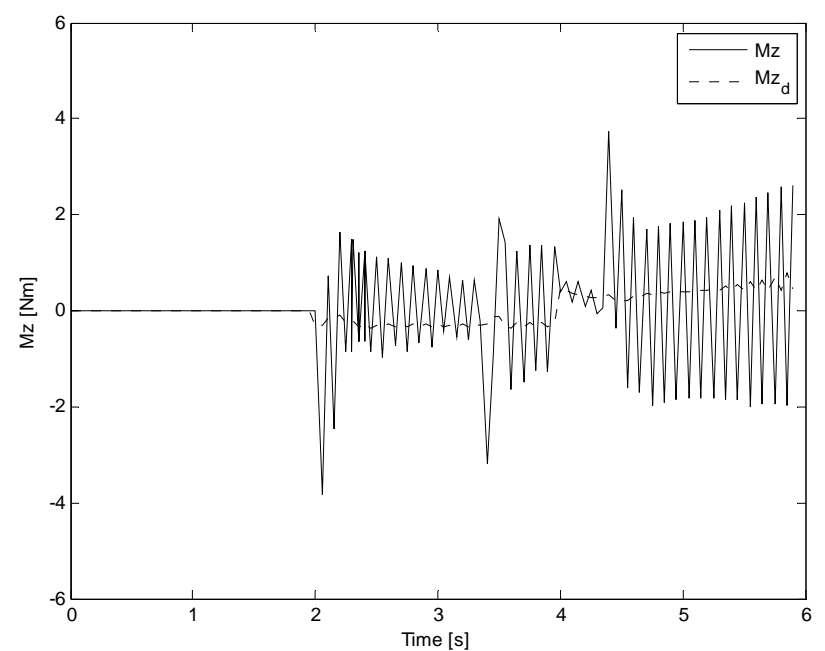

Figure 14. Case 3:Yaw Moment tracking, actual (solid) vs. desired (dashed).

\section{Conclusions}

A sophisticated and novel control algorithm is presented in the above. By design, it fully respects nonlinear tyre characteristics, is efficient enough to be executed in real-time, 
and appears general enough to be applied to a wide variety of vehicle manoeuvres and a large number of actuator combinations. This is expected to include so-called torque vectoring, when for example individual electric drive motors are used in addition to individual brake actuators. The analytical aspects of the control algorithm were motivated by a previous (QLOC) algorithm, used for post-impact path and speed control of a destabilized vehicle. The present work shows that the same general approach can be applied to limit handling manoeuvres without any associated yaw-sideslip instability. Future work is needed to compare performance to those of independent numerical optimizations, and of course further work is needed to implement this type of controller in experimental vehicles.

A secondary contribution of the present paper is to show that it is not always necessary to pre-define a target path (the limitations of which have been summarized above) in order to carry out limit handling manoeuvres. The lane-change manoeuvre considered in the present work determines a control action that coordinates (automated) steering and braking actuators. It is also feasible that (front) steering is determined directly by a human driver while the other actuators are used in support of the driver actions. The acceleration reference could, in principle be found from external information (e.g. radar reflections) to determine that the manoeuvre is needed (lane-change in this case). Or it could be found from a driver interpreter function, or it might be found from a combination of external sensing and driver interpretation, an intriguing problem not so far considered in the literature.

Future work is also needed to formulate the optimal particle motion in terms of coupled lateral and longitudinal accelerations: the 'fastest possible' lane change may not necessarily capture all relevant and robust safety criteria for collision avoidance, as suggested by Case 3, where the slower lane-change is due to the larger speed reductions compared to the other cases. And of course, experimental validation is also required.

\section{REFERENCES}

[Choi 2011] Choi, J. , Kim, K. and Yi, K. Emergency driving support algorithm with steering torque overlay and differential braking, in IEEE Conference on Intelligent Transportation Systems, Proceedings, ITSC, 2011, pp. 1433-1439.

[Dingle 2010] Dingle, P. and Guzzella, L. Optimal emergency maneuvers on highways for passenger vehicles with two- and four-wheel active steering, Am. Control Conf. (ACC), 2010, 2010.

[Distner 2007] Distner, M. and Bengtsson, M. City safety - A system addressing rear-end collisions at low speeds, pp. 1-7, 2007.

[Fredriksson 2004] Fredriksson, J., Andreasson, J. and Laine, L. Wheel force distribution for improved handling in a hybrid electric vehicle using nonlinear control, 2004 43rd IEEE Conf. Decis. Control (IEEE Cat. No.04CH37601), pp. 4081-4086 vol.4, 2004.

[Galvani 2014] Galvani, M. , Biral, F. , Nguyen, B. M. and Fujimoto, H. Four wheel optimal autonomous steering for improving safety in emergency collision avoidance manoeuvres, 2014 IEEE 13th Int. Work. Adv. Motion Control, pp. 362-367, Mar. 2014.

[Hayashi 2012] Hayashi, R., Isogai, J., Raksincharoensak, P. and Nagai, M. Autonomous collision avoidance system by combined control of steering and braking using geometrically optimised vehicular trajectory, Veh. Syst. Dyn., vol. 50, no. sup1, pp. 151-168, Jan. 2012.

[Kiencke 2005] Kiencke, U. and Nielsen, L. Automotive control systems: for engine, driveline, and vehicle: second edition. 2005, pp. 1-512.

[Longoria 2009] Longoria, R. G. Coordinated and reconfigurable vehicle dynamics control, IEEE Trans. Control Syst. Technol., vol. 17, no. 3, pp. 723-732, May 2009.

[Mechanical Simulation 2005] Mechanical Simulation Corporation, M. Ann Arbor, Carsim. [Online]. 2005. Available from $<$ www.carsim.com>

[Shah 2014] Shah, J. , Zegelaar, P. and Best, M. , Performance of emergency steer assist in front wheel and rear wheel steering vehicle, pp. 2228, 2014

[Shibata 2014] Shibata, N., Seiji, S., and Takahiro, W. , Collision avoidance control with steering using velocity potential field. Intelligent Vehicles Symposium Proceedings, 2014 IEEE. IEEE, 2014.

[Shim 2012] Shim, T., Adireddy, G. and Yuan, H. L., Autonomous vehicle collision avoidance system using path planning and modelpredictive-control-based active front steering and wheel torque control. Institute of Mechanical Engineers Proceedings, 2012. 2012, pp. 226(6): 767-778.

[Yang 2012] Yang, D. , Gordon, T. J., Jacobson, B. and Jonasson, M., Quasi-linear optimal path controller applied to post impact vehicle dynamics, IEEE Trans. Intell. Transp. Syst., vol. 13, no. 4, pp. 1586-1598, Dec. 2012.

[Yang 2014] Yang, D., Jacobson, B., Jonasson, M. and Gordon, T. J. Closed-loop controller for post-impact vehicle dynamics using individual wheel braking and front axle steering, Int. J. Veh. Auton. Syst., vol. 12, no. 2, pp. 158, 2014. 